\title{
Assessment of Casual Relations, Direct and Indirect Effects of Latent Covariates On CD4 Cell Count Change Among HIV Positive Adults
}

Awoke Seyoum Tegegne ( $\sim$ bisrategebrail@yahoo.com )

Bahir Dar University

\section{Research Article}

Keywords: Socio-demographic, individual characteristics, Structural equation, CD4count change

Posted Date: August 13th, 2021

DOl: https://doi.org/10.21203/rs.3.rs-780561/v1

License: (1) (1) This work is licensed under a Creative Commons Attribution 4.0 International License.

Read Full License 


\section{Abstract}

Background: The use of structural equation modelling and latent variables remains unusual in epidemiology despite its potential usefulness and assessment of casual relations. Measuring direct and indirect effect of latent variables helps for proper intervention and for the ART program to be effective. The main objective of current investigation was to assess casual relations, direct and indirect effect of latent covariates on CD4 cell count change for HIV positive adults under HAART.

Methods: Based on the repeated measures of CD4 cell count change data obtained in ART section at Felege Hiwot Teaching and Specialized hospital, AMOS software was employed parameter estimation. The data was conducted on 792 randomly selected HIV positive adults. The data was collected by the health staff for sake of delivering medication adherence.

Results: CD 4 cell count change was directly and indirectly affected by the latent variables. The powers of effects of observed variables with and without latent variables were a little bit different from each other. Hence, the power effect of observed variables with latent variables was lower as compared to without latent variables. The direct effect of latent variables on the response variable was a little bit greater than indirect effect.

Conclusion: The power of effects of observed variables were stronger than their effects with latent variables. Hence, the latent variables had significant contributions for progress of CD4 cell count change. Health related education about the direct and indirect effect of latent variables should be given for patients under HAART.

\section{Introduction}

The casual effect of a treatment on health progression is generally mediated by several intermediate variables. Estimation of the parameters related to the causal effect of a treatment that is mediated by a given intermediate variable is termed as the indirect effect of the treatment [1]. On the other hand, estimation of parameters when it is not mediated by intermediate variable is termed as the direct effect of the treatment[1]. Such relation is often relevant to the understanding and to the design of clinical and public health interventions. Estimation of the direct and indirect effect of a treatment on health progress is a crucial focus of epidemiological and clinical research and is major methodological advancements[2]. One of the well-known measure/ indicator of health progression rate under HAART adherence is CD4 cell count change.

To assess the various causal relations and effects, different authors proposed different approaches[3]. One of the approaches is presentation of a general locally efficient estimating function-based methodology for estimation of the corresponding causal parameters. Another approach is to identify the pathways by which a treatment is acting and to quantify the component of the treatment's effect with and without a given intermediate variable (the indirect and direct effects of the treatment) [2]. Estimation 
of the direct and indirect effects of a treatment helps in understanding of the treatment's action and to design clinical and public health interventions.

To compute the direct causal effect of treatment response, it is possible to assume existence of counterfactual outcomes for a randomly selected individual[2, 4]. The population direct effect is defined as the mean of these individual counterfactual direct effects $[5,6]$. On the other hand, in path analysis (structural equation modelling), one can see the direct, indirect and total effects of the latent variables and the model does not assume that change is constant and linear[5]. In path analysis a unique additional contribution of a latent variable has been determined[7, 8].

Identifying factors affecting the level of CD4 cell count change other than ART would help health professionals and patients to facilitate proper management and monitoring of the health care intervention on individuals with highest risk (lowest CD4 cell count)[9].

Previously, researches were conducted on predictors of CD4 cell count change using classical statistics (PROC GLM ANOVA) model. However, in the previous researches, the effect of latent variables on the variable of interest is not investigated[9]. Previously, the effect of observed variables namely age, weight, visiting times, baseline CD4 cell count, WHO stages, adherence and follow up visits on CD4 cell count change data is conducted using the same data by the same author using repeated measures ANOVA[10]. In such study, the effects of latent variables are not taken in to account.

The SEM analysis with PROC CALIS on the other hand, allowed for explicit representation of measurement error and provided more information than the repeated measures ANOVA with PROC GLM[10]. The purpose of this study was therefore, to investigate the casual relation, direct and indirect effect of latent variables on longitudinal repeated measures of CD4 cell count change for HIV positive adults under HAAR.

\section{Methods}

\section{Study area and Population}

The study was conducted in Amhara Region, North-West Ethiopia.

\section{Data source}

The data consists of retrospective longitudinal CD4 cell count data obtained from 792 randomly selected HIV Positive adults who registered for the first 10 months of 2012 and followed up to 2017( five years data). The study population belongs to HIV positive adults whose follow ups were from 2012 to 2017, end of study period.

\section{Study design}


The data used under current investigation consists of secondary data and a retrospective longitudinal study design was employed.

\section{Data collection procedures}

The data were collected by the health staff for primary interest of treatment follow ups. The ssecondary data obtained at ART section of the hospital also collected by the health staff in consultation with principal investigator.

\section{Quality of data}

This was conducted by data controllers in ART section of the hospital. The controllers were taken intensive training by the Ministry of Health for different purposes. The reliability of variables was assessed using Crombatch's alpha and the variables included in the study were tested for consistency of understanding and the completeness of the data items on 75 random samples. Necessary amendments were made on the final data collection sheet.

\section{Variables under study}

\section{Response Variable}

The longitudinal response variable for current study was CD 4 cell count change recorded at each visiting time. CD 4 cell count change was defined as the change/ or progress obtained at each visiting time which was obtained by considering the CD4 cell count at the current visit and the CD 4 cell count recorded immediately before the current visit.

\section{Predictor variables}

From medical records of each HIV positive patients, Social characteristics, individual behaviours, clinical factors, HAART adherence competence and retention in HAART medication were recorded. The association for predictor variables, taken from charts of patients is indicated in Fig. 1.

Structural Equation Model (SEM) was used in current investigation. Complex relationships were existed between CD4 cell count change and observed and latent variables. The casual relations, direct and indirect effect of latent variables on the variable of interest were assessed. Unlike conventional regression methods, SEM enables us to deal with such complex relationships and to estimate the direct and indirect effects of the variables of interest.

In current investigation; the variables such as religious affiliation, ethnicity, migration, that cannot be measured directly from patients' data were considered as socio-demographic latent variables. Economic factors such as access to drinking water, electric city, employment status not included in the data also considered as latent variables. Clinical factors like viral load progression, depression of patients because of medication, medication allergic and patient-health staff relation were considered as clinical latent variables. Similarly, individual factors such as doing physical exercise, refreshment of him/her, life 
experience, cognitive style, biological and genetically factors were considered as individual variables that cannot be measured directly and treated as latent variables.

Latent variables (socio-economic) was hypothesized to depend on education level, residence area, marital status, disclosure level of disease. The latent variable (economic factor) was hypothesized to depend on income level and ownership of cell phone, Individual characteristics was hypothesized to depend on weight, age, sex and clinical factors was hypothesized to depend on WHO stages, adherence level and baseline CD4 cell count. Similarly, the latent variable retention in HAART medication care was hypothesized to depend on follow up visits, socio-demographic factors, economic factors and individual characteristics. The latent covariates, HAART adherence competence was hypothesized to depend on clinical factors and retention HAART medication care. Finally, CD 4 cell count change was hypothesized to depend on retention in HAART medication care and HRRAT adherence competence directly and to be dependent indirectly on the other covariates.

In this study, the observed variables like age, weight, visiting times, baseline CD4 cell count were constructed on latent variables (Socio-demographic, economic, individual and clinical variables). Sociodemographic and clinical factors were also constructed to another latent variables, HAART medication care and real biological, psychological and HAART adherence competence. The retention in medication adherence and adherence competence were constructed to the variable of interest. The effect of exogenous variables on the variable of interest measured based on the estimates of parameters existed between the exogenous and endogenous variables (4). The effect of exogenous variables on the variable of interest also measured based on the amount of variance of errors created during estimation.

This was constructed using AMOS software version 22. The software computed the values of parameters, variance of error terms and disturbance terms $\left(D_{1}, D_{2}, . D_{6}\right)$.

Data analysis was carried out to obtain descriptive statistics (means and standard deviations (SD)) for each of the independent variables. Finally, using structural equation modelling, several trajectory analyses were conducted, with which we can specify the direct and indirect relationship among variables.

\section{Application of guidelines and regulations}

The study was performed in accordance with relevant guidelines and regulations.

\section{Results}

Among all the sample of 792 patients: $40.9 \%$ were rural residents; $50.6 \%$ were females; $56.3 \%$ were living with their partner; $33.6 \%$ disclosed their disease to family members, $49.2 \%$ were owners of cell phones, $25.5 \%$ were medication adherent, only $11.5 \%$ had high income and $20.6 \%$ had no education. The average (median) weight of all patients was 58kg (IQR: $(52,64)$ ), average years of all patients was 36 years (IQR: $(28,48))$. 
The estimate of regression coefficients was conducted using maximum likelihood estimation technique and indicated in hypothesised path analysis as shown in Fig. 1. Figure 1 revealed that $|\mathrm{C} . \mathrm{R}|=$ |Estimates/S.E| was greater than 1.96 for 0.05 level of confidence and greater than 2.56 for 0.01 level of confidence for all covariates and this further indicates that latent variables had direct and indirect effects on the CD 4 cell count change[11, 12].

Table 1

Structural Equation Model: Regression Coefficients

\begin{tabular}{|llll|}
\hline Variable & Estimates & Standard deviation & z-values \\
\hline literate & 0.810 & 0.900 & 25.321 \\
\hline With partner & 0.598 & 0.773 & 21.762 \\
\hline Urban & 0.614 & 0.783 & 22.042 \\
\hline Disclosed & 0.293 & 0.542 & 15.245 \\
\hline High income & 0.137 & 0.370 & 10.401 \\
\hline Owner of cell phone & 0.556 & 0.746 & 20.980 \\
\hline age & -73.283 & 75.138 & -27.445 \\
\hline Weight & 63.713 & 65.373 & 27.426 \\
\hline Male & -0.490 & 0.700 & -19.706 \\
\hline Baseline CD4 cell count & 157.028 & 187.476 & 23.570 \\
\hline Adherent & 0.330 & 0.574 & 16.166 \\
\hline notstage4 & -0.784 & 0.885 & -24.913 \\
\hline Visiting time & 11.445 & 13.203 & 24.393 \\
\hline Socio-demographic variables & 0.924 & 0.342 & 76.027 \\
\hline Economic factors & 0.874 & 0.825 & 24.811 \\
\hline Individual characteristics & 0.938 & 0.462 & 57.133 \\
\hline Clinical factors & 0.996 & 0.883 & 31.741 \\
\hline Retention in medication care & 0.654 & 0.742 & 24.803 \\
\hline HAART adherence competence & 0.725 & 0.834 & \\
\hline & & & 2462 \\
\hline
\end{tabular}

In the hypothetical relationships described in Fig. 1, non-recorded variables at each visiting time were categorized as socio-demographic, economic, individual characteristics and clinical factors. The 
magnitude of parameter estimation was conducted using AMOS version 22 and indicated in Fig. 1.

Figure1, indicates that socio-demographic factors had direct and significant effect on retention medication care (F5) with regression coefficient of 2.59 and p-value $=0.0021)$. Similarly, economic and individual factors had direct and significant effect on the retention medication care (F5) with regression coefficient of 2.08 for each with $p$-values $=0.0012 \& p$-value $=0.023$ respectively.

The latent variable retention medication care had significant and direct effect on another latent variables HAART adherence competence (F6) with regression coefficient of 2.31 , p-values $=0.0021$. Similarly, clinical factors also had direct and significant effect of HAART adherence competence (F6).

Finally, the latent variables, retention in medication care and HAART adherence competence had direct and significant effect on the variable of interest (CD4 cell count change).

The hypothetical relations in Fig. 1, also indicates that the latent variables namely, socio-demographic factors (F1), economic factors (F2), individual factors (F3) and clinical factors (F4) had indirect effect on the $\mathrm{CD} 4$ cell count change.

Figure 1 also indicates that the observed variables like level of education, residence area, marital status and disclosure of the disease to community living together had direct and significant effect on the latent variable, socio-demographic factors (F1). The observed variables, level of income and ownership of cell phone significantly affected economic factors (F2). Age and sex of patients affected significantly another latent variable (individual factors (F3)). Baseline CD4 cell count, WHO stages and adherence level significantly affected clinical factors. The observed covariate, follow up visits had significant effect on the latent covariates retention medication care (F5).

Hence, latent variables that can't be measured directly also had direct and indirect effect on CD4 cell count change.

In the estimation of the covariance structure, key indicators of goodness-of-fit provided that chi-square = 983.45, with $p$-value for chi-square $<0.01$, which indicates that the chi-square statistic is not closer to zero and the corresponding $p$-value is very small (significant), which is an indicator of weak fit. This indicates that the model is inadequate. However, RMSEA was estimated to be $0.01, \mathrm{CFI}=0.97$, Non-normed fit index $(\mathrm{NNFI})=0.96$ and NFI $=0.95$. Hence, RMSEA, CFI and NFI assured for the model to be good fit. The chisquare probability for current repeated data indicated an unacceptable model fit (chi-square $=9.101, \mathrm{df}=$ $3, p=0.028)$.

The difference between observed and expected covariance structure matrices under current investigation was measured using chi-square value. The model under the study was also determined using a chisquare and the value was close to zero and corresponding probability value was greater than 0.05 . Hence, the value for RMSEA was 0.123 which indicates that the model was unacceptable. On the other hand, the value for CFI was 0.998 and the corresponding value of NNFI was 0.988 which indicates that the model was acceptable to fit the data in current investigation with a value greater than $0.90[13,14]$. 


\section{Direct and Indirect effect of latent variables}

The latent covariates, Socio-demographic, economic, individual, clinical factors, retention in HAART medication care and HAART adherence competence had direct and indirect effect on the CD4 cell count change. The direct and indirect effects are indicated in Table 2.

Table 2

Direct and indirect effect of latent covariates on CD4 cell count change

\begin{tabular}{|lllll|}
\hline \multirow{2}{*}{ Effect } & \multicolumn{2}{l}{ Direct effect } & \multicolumn{2}{l|}{ Indirect effect } \\
\cline { 2 - 5 } & Estimate & Standard error & Estimates & Standard errors \\
\hline Socio-demographic variables & 0.854 & 0.341 & 0.421 & 0.0321 \\
\hline Economic factors & 0.642 & 0.453 & 0.405 & 0.4525 \\
\hline Individual characteristics & 0.728 & 0.432 & 0.352 & 0.4216 \\
\hline Clinical factors & 0.526 & 0.273 & 0.435 & 0.2723 \\
\hline HAART adherence competence & 0.832 & 0.543 & 0.654 & 0.5434 \\
\hline Retention in HAART medication care & 0.725 & 0.546 & 0.643 & 0.5463 \\
\hline
\end{tabular}

Table 2 indicates that effect of latent variables which belongs to direct effect was a little bit greater than indirect effects. Hence, CD 4 cell count change had been affected by direct and indirect involvement of latent variables.

\section{Comparison of predictors of CD4 cell count change with and without latent variables}

The comparison of approaches was conducted considering latent variables and without latent variables. Figure 1 indicates that, some of the variables are manifest and the others are latent. To compare approaches, the effect of observed variables without latent was compared with latent variables. The effect of manifest variables like level of education, marital status, sex of patients, disclosure of the disease to community living together, ownership of cell phone, level of income, weight, age, sex of patients, baseline CD4 cell count, WHO stages, adherence level and visiting times were potential predictors of CD 4 cell count change without any latent variables using the same data and same study area. Here, it was possible to compare the effect of these manifest variables with and without latent variables, conducted previously by the same author, data and same study area[12,15].

A baseline measurement and its change had different residual errors. The measurement model for each sex were verified and the result in the loadings and the fit indices were approximately similar while parameterization with and without equality of constraints. 
A combination of repeated measurements was considered by the latent variable analysis and the result makes the latent variables vary together. Hence, the individual measurement analysis gave the same conclusion which was not achieved in the analysis conducted without latent variable approach. This implies that whenever, the individual analyses are not consistent, a latent variable model provides an easy interpretable synthesis.

\section{Discussion}

In current investigation, the association of socio-demographic, individual characteristics and clinical variables were investigated using SEM (path analysis). The result in this investigation revealed that Socio-demographic factors, economic factors and individual characteristics have direct and significant association with retention in HAART program. This findings is agreed with one of the previous investigation[16].

Therefore, socio-demographic disadvantageous HIV positive adults are related with lower CD4 cell count change[17]. Education is to a large extent correlated with socio-demographic and much of the complexity that is evident in the relationship between income and HIV prevalence is also evident in the relationship between education and HIV prevalence. Hence, relatively uneducated patients are less likely to know what AIDS is and how HIV is transmitted from one to another[17].

The relationship between marital status and HIV infection is complex. Hence, the risk of HIV prevalence remained significantly high among unmarried compared with married people when only sex behaviour factors are controlled for the given model[18].

The adherence level of patients is highly associated with socio-demographic characteristics such that patients disclosed their disease to the community living with them had good competence level of food and medication adherence [19]. Thus, it is said that a person disclosed the disease to people living together may get social support from the community and might have good medication adherence and this further leads improvement of the CD4 cell count change in successive visits[20].

The social support given to HIV patients who disclosed their disease helps to have very good retention in HIV medication care which is a crucial activity for achieving long term survival with the virus[21].

Similarly, Clinical factors such as baseline CD4 cell count, WHO stages and adherence level had direct and significant effect on HAART adherence competence. Hence, HIV positive adults with good adherence level, high baseline CD4 cell count and WHO stages of I had good HAART adherence competence. This result is similar with previously conducted research[22]. Retention in medication care had also direct and significant effect on HAART adherence competence which means patients which closely follow their prescribed medication given by the health staff had good adherence competence. This result agreed with previous researches $[23,24]$. Finally, retention in medication care and adherence competence had direct and significant effect on the variable of interest (CD4 cell count change) which has similar argument with previous researches[24]. 
The economic factors such as patients with cell phone and those who had high income associated with high retention in the medication care. Hence, patients with high income may use different alternatives to get pills and he/she also uses proper food adherence schedules for the treatment to be effective and this encourages the patient to attend the visits of health institution[22]. Cell phone of patients can play significant role in taking pills on time and to remind the date that the patient should visit the hospital and this has indirect effect on the status of CD4 cell count change. Cell phone helped patients to be HAART adherent because of its alarm (memory aid) for reminding the time pills are taken[25]. This finding is consistent with findings from another study[26] and suggests the need for making cell phones available to the needy HAART attendants. This finding is also consistent with previously conducted research [27].

Clinical factors such as patients' CD 4 cells count significantly affected their retention of medication care. Patients with high number of CD4 cell count encouraged the patient to be HAART adherent as compared to patients with less number of CD4 cell count[28, 29]. This result indicates that clinical factors (CD4 cell count and WHO stages) positively associated with retention of medication care.

As a conclusion, latent variable and structural equation models enabled us to present synthetic results rather than separate analyses for each sex group and to perform a detailed analysis of the causal mechanisms involved. Poor adherent patients had low results in the variable of interest which indicates that adherence to HAART and CD4 cell count change are positively correlated to each other. Patients with good performance of adherence to medication had better CD4 cell count change. The current study indicates that the variable of interest (CD4 cell count change) increased over time. However, its progress was different for different groups[30].

When a single measurement exhibits a relationship with the latent variable, considering several measurements have much gain and a latent variable are recommended rather than separate analyses of each indicator[31]. Structural equation and path analyses are very useful for causal interpretation[32]. Of course, the interpretations are conditional on the validity of the assumed model[3].

Single intervention strategy cannot improve risks of HIV patients. The result in current investigation corroborates key factors like realistic assessment of patients' knowledge/level of education and understanding of the regimen, residence area of patients, age and sex of patients. The research endeavoured to identify certain groups that require special attention; and this helps to intervene in HAART program to be effective and elongate patients` lives with the virus.

Moreover, interventions need to be designed to promote early HIV testing and early enrollment of HIV infected individuals into ART services. Health related issues about socio-demographic, economic, individual characteristics and clinical factors, improving public awareness through advocacy and social mobilization should be included in the ART service. It is strongly recommended that underline the need for ART in HIV infected patients for immune reconstitution.

\section{List Of Abbreviations}


RMSEA

Root Mean Square Error Analysis, HAART = Highly Active antiretroviral therapy, LGM = Latent Growth Model, CLGM = Covariate Latent Growth Model, HIV = human immunodeficiency virus, SEM = Structural Equation Modeling, CFI = Comparative Fit Indices,

\section{Declarations}

Consent for participants: Since the data was secondary and there was no chance of getting participants, consent for participants was not obtained from respondents. However, to get the secondary data from the hospital in the study area, Ethical clearance certificate had been obtained from two universities namely Bahir Dar University, Ethiopia with Ref RCS/1412/20012. I can attach the ethical clearances certificate up on request.

Competing interests. As no individual or institution funded this research, there is no conflict of financial and non-financial interest between authors or between authors and institutions.

Funding: Not applicable

Authors' contributions: The first and corresponding author wrote the proposal, develop data collection format, supervise the data collection process, analysed and interpreted the data.

Availability of data and materials: I confirm that the data used for this study is available at corresponding authors and can be submitted upon request.

Consent for publication: This manuscript has not been published elsewhere and is not under consideration by any other journal. All authors approved the final manuscript and agreed with its submission to this journal. We agreed about authorship and order of authors for this manuscript.

\section{References}

1. Beyene, M. B. \& Beyene, H. B. Predictors of late HIV diagnosis among adult people living with HIV/AIDS who undertake an initial CD 4 T cell evaluation, Northern Ethiopia: a case-control study. PloS one, 10 (10), 0140004 (2015).

2. Tegegne, A. S. \& Zewotir, T. Determinants of CD4 cell count change and time-to default from HAART; a comparison of separate and joint models. BMC infectious diseases, 18 (1), 197 (2018).

3. Adams, M. \& Luguterah, A. Longitudinal analysis of change in CD4 + cell counts of HIV-1 patients on antiretroviral therapy (ART) in the Builsa district hospital.European Scientific Journal, 2013. 9(33).

4. Muthén, B. \& Asparouhov, T. Causal effects in mediation modeling: An introduction with applications to latent variables. Structural Equation Modeling: A Multidisciplinary Journal, 22 (1), 12-23 (2015). 
5. Katunarić, V. Od egalitarnog sindroma do izvrsnosti: O načinima legitimiranja društvenih nejednakosti. Politička misao, 48 (03), 11-34 (2011).

6. Louizos, C. et al. Causal effect inference with deep latent-variable models. in Advances in Neural Information Processing Systems. 2017.

7. Cheung, G. W. \& Lau, R. S. Testing mediation and suppression effects of latent variables: Bootstrapping with structural equation models. Organizational research methods, 11 (2), 296-325 (2008).

8. Lleras, C. Path analysis. Encyclopedia of social measurement, 3 (1), 25-30 (2005).

9. Asfaw, A. et al. CD4 cell count trends after commencement of antiretroviral therapy among HIVinfected patients in Tigray, Northern Ethiopia: a retrospective cross-sectional study. PloS one, 10 (3), 0122583 (2015).

10. Guerrero, J. M. et al. Hierarchical control of droop-controlled AC and DC microgrids-A general approach toward standardization. IEEE Transactions on industrial electronics, 58 (1), 158-172 (2010).

11. Marsh, H. W. \& Hocevar, D. Application of confirmatory factor analysis to the study of self-concept: First-and higher order factor models and their invariance across groups. Psychological bulletin, 97 (3), 562 (1985).

12. MacCallum, R. C. \& Austin, J. T. Applications of structural equation modeling in psychological research. Annual review of psychology, 51 (1), 201-226 (2000).

13. Hoyle, R. H. The structural equation modeling approach: Basic concepts and fundamental issues. 1995.

14. Roth, D. L. et al. Life events, fitness, hardiness, and health: a simultaneous analysis of proposed stress-resistance effects. Journal of Personality and Social Psychology, 57 (1), 136 (1989).

15. Duncan, T. E. et al. Latent variable modeling of longitudinal and multilevel substance use data. Multivar. Behav. Res, 32 (3), 275-318 (1997).

16. Hu, L. \& Bentler, P. M. Cutoff criteria for fit indexes in covariance structure analysis: Conventional criteria versus new alternatives. Structural equation modeling: a multidisciplinary journal, 6 (1), 1-55 (1999).

17. Bayou, T. et al. Factors determinant for change of initial antiretroviral treatment regimen among patients on ART follow-up clinic of Mekelle Hospital, Mekelle, Ethiopia. Int J Basic Clin Pharmacol, 3 (1), 44-49 (2014).

18. Do, N. T. et al. Psychosocial factors affecting medication adherence among HIV-1 infected adults receiving combination antiretroviral therapy (CART) in Botswana. AIDS research and human retroviruses, 26 (6), 685-691 (2010).

19. Langebeek, N. et al. Predictors and correlates of adherence to combination antiretroviral therapy (ART) for chronic HIV infection: a meta-analysis. BMC medicine, 12 (1), 1-14 (2014). 
20. Nosyk, B. et al. Characterizing retention in HAART as a recurrent event process: insights into 'cascade churn'. AIDS (London, England), 2015. 29(13): p. 1681.

21. Schwimmer, J. B., Burwinkle, T. M. \& Varni, J. W. Health-related quality of life of severely obese children and adolescents. Jama, 289 (14), 1813-1819 (2003).

22. Ezeamama, A. E. et al. Age, sex, and nutritional status modify the CD4 + T-cell recovery rate in HIVtuberculosis co-infected patients on combination antiretroviral therapy. International Journal of Infectious Diseases, 35, 73-79 (2015).

23. Sagarduy, J. L. Y. et al. Psychological model of ART adherence behaviors in persons living with HIV/AIDS in Mexico: a structural equation analysis. Revista de saude publica, 51, 81 (2017).

24. Rao, D. et al. A structural equation model of HIV-related stigma, depressive symptoms, and medication adherence. AIDS and Behavior, 16 (3), 711-716 (2012).

25. Ye, R. et al. Studies on the determinants and changes related to the natural CD4 (+) T cell counts among antiretroviral treatment-naive HIV/AIDS patients in Dehong prefecture, Yunnan province. Zhonghua liu xing bing xue za zhi = Zhonghua liuxingbingxue zazhi, 2011. 32(9): p.882-887.

26. Kipp, A. M. et al. Socio-demographic and AIDS-related factors associated with tuberculosis stigma in southern Thailand: a quantitative, cross-sectional study of stigma among patients with TB and healthy community members. BMC Public Health, 11 (1), 675 (2011).

27. Maqutu, D. et al. Determinants of optimal adherence over time to antiretroviral therapy amongst HIV positive adults in South Africa: a longitudinal study. AIDS and Behavior, 15 (7), 1465-1474 (2011).

28. Florence, E. et al. Factors associated with a reduced CD4 lymphocyte count response to HAART despite full viral suppression in the EuroSIDA study. HIV medicine, 4 (3), 255-262 (2003).

29. Amberbir, A. et al. Predictors of adherence to antiretroviral therapy among HIV-infected persons: a prospective study in Southwest Ethiopia. BMC public health, 8 (1), 265 (2008).

30. Ebonyi, A. O. et al. Factors associated with a low CD4 count among HIV-1 infected patients at enrolment into HAART in Jos, Nigeria. 2014.

31. Montarroyos, U. R. et al. Factors related to changes in CD4 + T-cell counts over time in patients living with HIV/AIDS: a multilevel analysis. PloS one, 9 (2), 84276 (2014).

32. Smith, C. J. et al. Factors influencing increases in CD4 cell counts of HIV-positive persons receiving long-term highly active antiretroviral therapy. The Journal of infectious diseases, 190 (10), 18601868 (2004).

33. Authors ' contributions: The first and corresponding author wrote the proposal, develop data collection format, supervise the data collection process, analysed and interpreted the data.

\section{Figures}




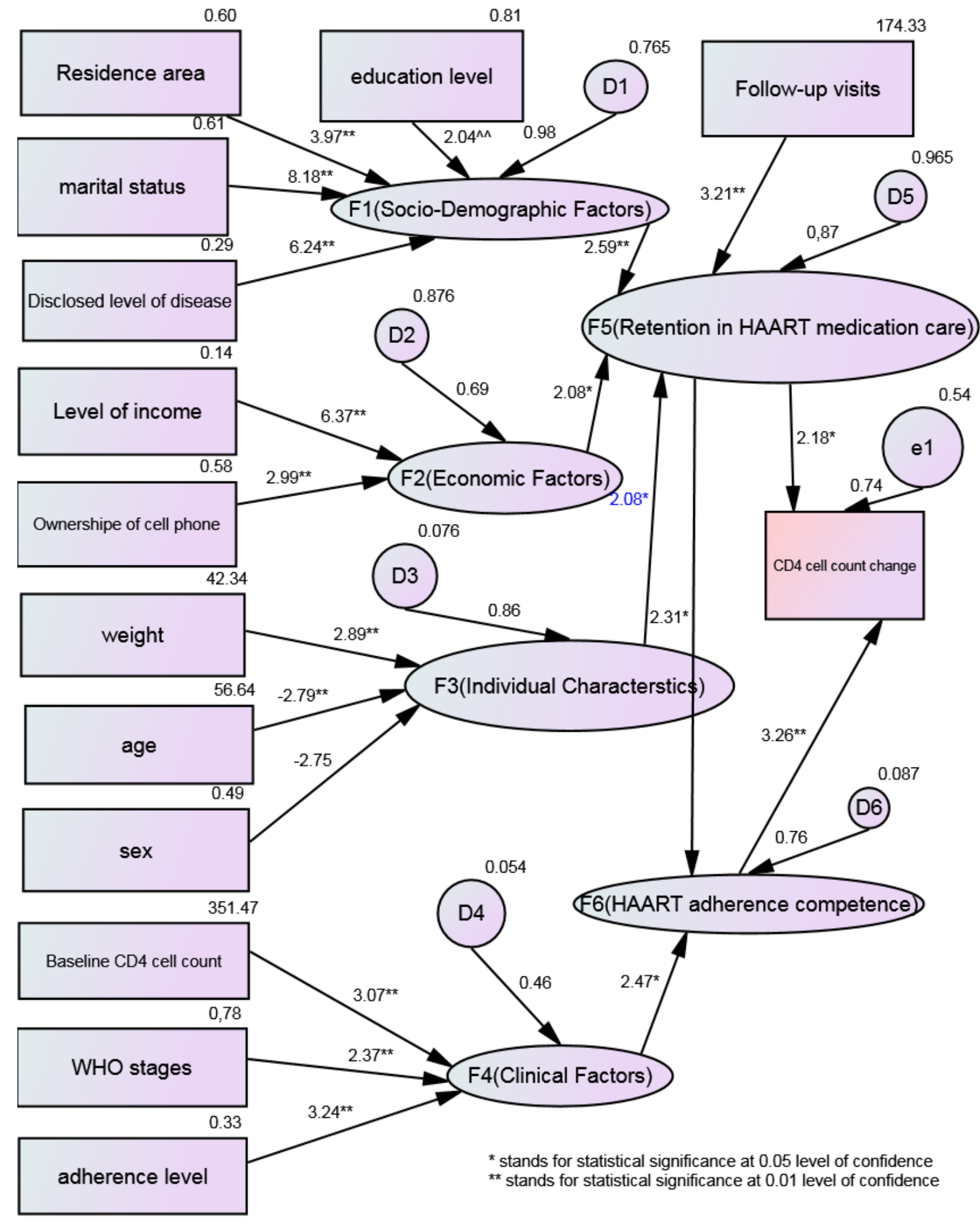

\section{Figure 1}

Path Analysis of predictor variables for CD4 cell count change after estimates of parameters and error terms 